\title{
PROGRESSION FROM ISOLATED GROWTH HORMONE DEFICIENCY TO COMBINED PITUITARY HORMONE DEFICIENCY
}

Manuela Cerbone, Mehul T Dattani

Developmental Endocrinology Research Group, UCL Institute of Child Health and Department of Endocrinology, Great Ormond Street Hospital for Children, WC1N 1EH London, UK

\section{Corresponding author:}

Mehul T Dattani

\section{Abstract:}

Growth hormone deficiency (GHD) can present at any time of life from the neonatal period to adulthood, as a result of congenital or acquired insults. It can present as an isolated problem (IGHD) or in combination with other pituitary hormone deficiencies (CPHD). Pituitary deficits can evolve at any time from GHD diagnosis. The number, severity and timing of occurrence of additional endocrinopathies are highly variable.

The risk of progression from IGHD to CPHD in children varies depending on the etiology (idiopathic vs organic). The highest risk is displayed by children with abnormalities in the Hypothalamo-Pituitary (H-P) region. Heterogeneous data have been reported on the type and timing of onset of additional pituitary hormone deficits, with TSH deficiency being most frequent and Diabetes insipidus the least frequent additional deficit in the majority, but not all, of the studies. ACTH Deficiency may gradually evolve at any time during follow-up in children or adults with childhood onset IGHD, particularly (but not only) in presence of H-P abnormalities and/or TSH Deficiency. Hence there is a need in these patients for lifelong monitoring for ACTH deficiency. GH treatment unmasks central hypothyroidism mainly in patients with organic GHD, but all patients starting GH should have their thyroid function monitored closely. Main risk factors for development of CPHD include organic etiology, H-P abnormalities (in particular pituitary stalk abnormalities, empty sella and ectopic posterior pituitary), midline brain (corpus callosum) and optic nerves abnormalities, genetic defects and longer duration of follow-up.

The current available evidence supports longstanding recommendations for the need, in all patients diagnosed with IGHD, of a careful and indefinite follow-up for additional pituitary hormone deficiencies.

\section{Highlights:}

- This review commences with a general overview on isolated GH deficiency and the current recommendation on monitoring for the risk of development of additional hormonal deficiencies.

- The evidence pertaining to the risk of progression from IGHD to CPHD and on the type and timing of additional pituitary hormone deficiencies is then summarized.

- The risk of developing central hypoadrenalism in adults with childhood-onset GHD and central hypothyroidism after the start of $\mathrm{GH}$ therapy is also discussed.

- Finally, the main risk factors associated with development of CPHD are evaluated.

Key words: Isolated growth hormone deficiency, Evolution, Combined pituitary hormone deficiency

This research did not receive any specific grant from funding agencies in the public, commercial, or not-forprofit sectors. 
Growth hormone deficiency (GHD) can present at any time of life from the neonatal period to adulthood, and can be a result of congenital or acquired insults.

In congenital cases, GHD can be found in association with brain and midline facial abnormalities or can be due to mutations in genes involved in hypothalamo-pituitary (H-P) development or in the GHRH/GH pathway. Acquired causes include trauma, central nervous system (CNS) infections, H-P tumours, infiltrative or granulomatous diseases, cranial irradiation, surgery or infarction of the H-P region. Patients in whom a clear etiology cannot be identified are considered "idiopathic". The latter category accounts for most individuals with childhood-onset GHD, whereas it is exceptionally rare in adults [1].

Short stature with a poor growth velocity may be the only clinical presenting feature of GHD in childhood. Other key features in the history and physical examination include: 1) in the neonate: hypoglycaemia, prolonged jaundice, microphallus with undescended testes, or traumatic delivery; 2) a history of cranial irradiation; 3) a history of head trauma or CNS infection; 4) consanguinity and/or an affected family member; and 5) craniofacial midline abnormalities [2]. When occurring after completion of linear growth, GHD can be more difficult to identify. Adult patients at risk of GHD and therefore needing testing include 1) those with signs and symptoms of $\mathrm{H}-\mathrm{P}$ disease, 2 ) those who have received cranial irradiation or treatment

for tumours, 3) those with traumatic brain injury or subarachnoid hemorrhage [3]. Additionally, clinical features of $\downarrow$-angerhans cell histiocytosis and sarcoidosis may be present and indicate a need for testing of the HP axis.

It is well-known that GHD can present as an isolated problem (IGHD) or in combination with other pituitary hormone deficiencies (CPHD). Pituitary deficits can evolve at any time from GHD diagnosis. The number, severity and timing of occurrence of additional endocrinopathies are highly variable.

Current paediatric guidelines state that, in patients with an initial diagnosis of IGHD, particularly those with

| an ectopic posterior pituitary or other developmental abnormalities, the clinician should be alert to the risk of the development of CPHD [2]. Similarly, adult guidelines suggest that thyroid and adrenal function should be monitored during GH therapy [1].

However, no specific recommendations exist on the process of measurement (eg baseline vs dynamic tests) and on the frequency of anterior pituitary function monitoring in patients with IGHD. Most paediatric endocrinologists routinely obtain thyroid function tests on all poorly growing children regardless of their $\mathrm{GH}$ status. However, the decision whether to evaluate the other axes in all children with IGHD varies between centres and clinicians.

The aim of this review is to summarize the current evidence on the progression from IGHD to CPHD in childhood, adolescence and adulthood. The review will particularly focus on the type of additional pituitary deficiencies, on the timing of occurrence and on the risk factors for the evolution of endocrinopathies.

\section{Risk of progression from IGHD to CPHD and type and timing of additional pituitary deficits}

The results from the main studies analyzing the risk of progression from IGHD to CPHD are summarized in Table 1.

In a large prospective, multinational study (GeNeSIS), data were analyzed in 5805 paediatric patients with idiopathic IGHD. CPHD developed in $2.0 \%$ in the overall cohort, and in $5.5 \%$ among children followed for a minimum of 3.5 years. The relative frequency of additional deficiencies was TSH > LH/FSH > ADH > ACTH. Median interval from diagnosis of GHD to additional pituitary hormone deficiency was 1.9 years for TSH, 2.4 years for both ADH and ACTH, and 3.3 years for LH/FSH [4]. In 716 paediatric patients from the same database with IGHD due to organic causes, there was a higher frequency of CPHD $(9.9 \%$ in the overall cohort, and $20.7 \%$ in the subgroup followed up for a minimum of 3.5 years). The most frequent additional deficiencies were TSH and LH/FSH [5]. In a retrospective study of 83 patients initially diagnosed as IGHD in childhood, 45\% developed CPHD after a median follow-up of 5.4 years. The authors suggested that the higher prevalence of evolution from IGHD to CPHD, as compared to previous reports, was partially explained by the longer duration of follow-up and by the single centre nature of the study with routine clinical and laboratory assessments increasing the sensitivity of detection of new hormone deficiencies. LH and FSH deficiencies (38\%) were the most common additional pituitary deficits, followed by TSH (31\%),

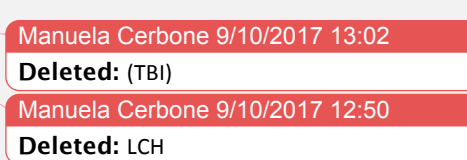

Deleted: $\mathrm{LCH}$

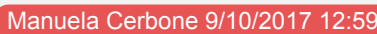

Deleted: (EPP)

Manuela Cerbone 9/10/2017 13:00

Deleted: (AP)

Manuela Cerbone 9/10/2017 12:47

Formatted: Font:Bold, Underline

Manuela Cerbone 9/10/2017 12:47

Formatted: Normal, No bullets or numbering 
ACTH (12\%) and ADH deficiency (5\%). ADH deficiency (3.1 \pm 1 years from GHD diagnosis) presented earlier and ACTH deficiency $(9.3 \pm 3.5$ years) presented later during follow up compared to LH/FSH $(8.3 \pm 4$ years) and TSH (7.5 \pm 5.6 years) deficiencies. Since half of the patients with IGHD developed the second hormone deficiency 5 years after the IGHD diagnosis, the authors reinforced the need for life-long monitoring of pituitary function in these patients [6].

Several authors have focused on the progression from IGHD to CPHD in patients with H-P abnormalities.. A recent large international database confirmed the value of classification of GHD patients by the presence and type of $\mathrm{H}-\mathrm{P}$ imaging abnormalities. Thirty-five percent of patients with $\mathrm{H}-\mathrm{P}$ structural abnormalities developed additional hormonal deficiencies and, when compared with patients with idiopathic GHD, they had more severe endocrine phenotypes [7]. Similarly, in a study of 103 patients, 72 of whom had IGHD and 31 had CPHD, structural H-P abnormalities were observed in $48.6 \%$ patients with IGHD and $93.5 \%$ with CPHD. Over a mean follow-up period of 4.5 years, $5.4 \%$ of subjects with an abnormal MRI and none of those with a normal MRI progressed from IGHD to CPHD. Amongst CPHD patients, an ectopic posterior pituitary/absent pituitary stalk was present in $80 \%$ of subjects with associated hypocortisolism \pm hypothyroidism as compared to $18.2 \%$ of subjects with hypogonadism [8]. In a retrospective longitudinal study of 67 patients with pjtuitary stalk jnterruption syndrome (absent or thin pituitary stalk, absent or hypoplastic anterior pituitary Jobe and/or ectopic posterior pituitary lobe) all patients had GHD at diagnosis. CPHD was found in all patients presenting in the neonatal period but only in $34 \%$ of the patients presenting later in life. CPHD in the neonatal group was complete (i.e., associated GH, ACTH, TSH and LH/FSH deficiencies) in 9 of 10 patients. This severe phenotype was related to severely abnormal pituitary development (i.e., anterior pituitary lobe not visible). In 21 patients presenting with growth retardation followed until final height attainment, the authors were able to document a progressive worsening of endocrine function in $44.6 \%$ of the cases (follow-up: 7.3 - 16.6 years). Re-evaluation at completion of growth of these 21 patients showed that 17 patients (81\%) had CPHD; this frequency was no longer different from that of neonatal patients. In summary, in contrast to patients diagnosed in the neonata period, who presented with complete hypopituitarism at diagnosis, most of the patients diagnosed after investigation for growth retardation presented a progressive impairment of the residual pituitary function throughout childhood justifying periodic monitoring for additional hormonal deficiencies in patients with pituitary stalk interruption syndrome [9]. Similar results were found in two other studies in children with pituitary stalk interruption syndrome, In the first study of 35 children with GHD and pituitary stalk interruption syndrome, GHD was isolated in $40.6 \%$ and combined with other pituitary deficiencies in $10 \%$ of patients below 11 years of age but CPHD was consistent at completion of growth in all patients [10]. In the second study on 83 children with pituitary stalk interruption syndrome, all patients had GHD, 79.5\% had deficiency in TSH, 67.5\% in ACTH, 65.1\% in LH/FSH, and $14.5 \%$ in PRL. IGHD was found in only $6 \%$ of patients [11]. Among 26 children and young adults with pituitary stalk dysgenesis (range at diagnosis: 2-27 years), at first assessment, $92.3 \%$ of patients had GHD, $26.9 \%$ had CPHD and $7.6 \%$ panhypopituitarism. Hormone deficiencies were progressive during follow-up in $84.6 \%$; $96 \%$ progressed to multiple pituitary hormone deficiencies and $46 \%$ to panhypopituitarism [12]. In another study, the gradual loss of anterior pituitary function was evaluated in 28 children with an invisible $(n: 18)$ or thin ( $n: 10)$ pituitary stalk on MRI. All the patients had GHD upon initial evaluation. At their last visit (ages: 10 to 24 years), 24 patients had TSH deficiency, 18 ACTH deficiency, 19 gonadotropin deficiency, and 3 Diabetes

| Insipidus (DI). An ectopic posterior pituitary lobe was present in 23 patients and the anterior pituitary, was judged to be absent or small in 20 of the patients [13]. In a recent large (n:8230) international pediatric database (GeNeSIS) additional hormonal deficiencies were found in $37.5 \%$ of patients with structural abnormalities, $47.5 \%$ of patients with known genetic defects (TSH >ACTH >LH/FSH>ADH) vs $3.8 \%$ in the idiopathic cases [7]. In a 2 year prospective pediatric study, authors analyzed data from 13 young | adults with childhood-onset GHD associated with ectopic posterior pituitary and "normal" GH response at the time of first assessment of $\mathrm{GH}$ secretion (GH peak after stimulation test between 5 and $10 \mu \mathrm{g} / \mathrm{l}$ ). Two | years after the retesting, eight of 13 patients (61\%) developed additional anterior pituitary deficits. Adrenal insufficiency was found in five patients, central hypothyroidism was diagnosed in four, and hypogonadotropic hypogonadism in one [14]

Finally, in a large observational prospective study (KIMS study) on 165 adults with severe IGHD due to | organic causes (malignancies or traumatic brain injury), new deficiencies were observed in $35 \%$ of the

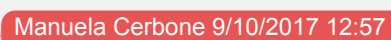

Deleted: EPP

Manuela Cerbone 9/10/2017 13:07

Deleted: (PSA)

Manuela Cerbone 9/10/2017 13:06

Deleted: P

Manuela Cerbone 9/10/2017 13:06

Deleted: S

Manuela Cerbone 9/10/2017 13:06

Deleted: I

Manuela Cerbone 9/10/2017 13:06

Deleted: S

Manuela Cerbone 9/10/2017 13:06

Deleted: (PSIS)

Manuela Cerbone 9/10/2017 13:00

Deleted: A

Manuela Cerbone 9/10/2017 13:00

Deleted: $P$

Manuela Cerbone 9/10/2017 13:00

Deleted: (AP)

Manuela Cerbone 9/10/2017 12:57

Deleted: EPP

Manuela Cerbone 9/10/2017 13:00

Deleted: AP

Manuela Cerbone 9/10/2017 13:06

Deleted: PSIS

Manuela Cerbone 9/10/2017 13:06

Deleted: PSIS

Manuela Cerbone 9/10/2017 13:06

Deleted: PSIS

Manuela Cerbone 9/10/2017 13:07

Deleted: PSIS

Manuela Cerbone 9/10/2017 13:09

Deleted: (PSD)

Manuela Cerbone 9/10/2017 13:09

Deleted: PSD

Manuela Cerbone 9/10/2017 13:00

Deleted: AP

Manuela Cerbone 9/10/2017 12:52

Deleted: PS

Manuela Cerbone 9/10/2017 12:57

Deleted: EPP

Manuela Cerbone 9/10/2017 13:00

Deleted: AP

Manuela Cerbone 9/10/2017 12:57

Deleted: EPP

Manuela Cerbone 9/10/2017 13:00

Deleted: AP

Manuela Cerbone 9/10/2017 13:02

Deleted: TBI 
patients, occurring up to 11 years from initial assessment. Secondary hypothyroidism was the most often recorded new deficiency followed by secondary hypogonadism and secondary hypoadrenalism, whereas ADH deficiency did not evolve in IGHD [15].

To summarize, the risk of progression from IGHD to CPHD in children varies depending on the etiology (5.5\% idiopathic vs $20.7 \%$ organic). The highest risk is displayed by children with abnormalities in the H-P region and ranges between $35 \%$ when considering all the $\mathrm{H}-\mathrm{P}$ abnormalities (including small anterior pituitary), $85 \%$ in cases with pituitary stalk dysgenesis, and $45-100 \%$ in children with pituitary stalk interruption syndrome, Interestingly, development of additional pituitary deficiencies was documented also in $61 \%$ of young adults with childood onset GHD due to ectopic posterior pituitary and a normal GH response to Insulin Tolerance Test (ITT) at time of retesting. Finally, the prevalence of progression from IGHD to CPHD in adult-onset organic severe GHD, has been reported as $35 \%$ (higher than in children with organic GHD (20.7\%) but lower than in children with H-P abnormalities (35-100\%)).

Heterogeneous data have been reported on the type and timing of onset of additional pituitary hormone deficits. This heterogeneity can probably be explained by the different populations included in the studies in terms of GHD etiology (organic vs idiopathic), age at GHD diagnosis (pediatric vs adult) and duration of follow-up (short vs long term follow-up). TSH deficiency was the most frequent and DI the least frequent additional deficit in the majority of the studies, whereas the prevalence of ACTH and LH/FSH deficiencies was variable. Inconsistent data have been reported in the two studies documenting the timing of evolution of endocrinopathies with TSH being the earliest and LH/FSH the latest deficit in one study and DI the earliest and ACTH the latest in another study. This heterogeneity could be partially explained by the different age and length of follow-up of the populations studied, but could also genuinely reflect the highly variable endocrine phenotype displayed by patients with CPHD.

\section{Childhood-onset IGHD and risk of central hypoadrenalism in adulthood}

Few studies focused on the risk, in patients with childhood-onset GHD, of developing central hypoadrenalism later in life and therefore on the need, in these patients, for lifelong monitoring for ACTH deficiency.

In a cross-sectional study assessing the relationship between GH, TSH and ACTH deficiency in 236 patients with childhood-onset idiopathic GHD, nine were found to have ACTH deficiency (4\%) and 21 (9\%) TSH deficiency. All of the ACTH-deficient subjects were also TSH deficient. Eight of the nine ACTH-deficient

| subjects had a midline abnormality on MRI (ectopic posterior pituitary or interrupted stalk). The authors concluded that ACTH deficiency was highly unlikely in the setting of normal pituitary anatomy [16]. Some sporadic cases have been reported of patients with IGHD developing ACTH deficiency during adult life. However, in keeping with the results from the previous study, all of these patients had either TSH deficiency or a gross anatomical abnormality of the H-P region $[17,18]$. Conversely, in another crosssectional study, the authors documented a significant risk of adult adrenal insufficiency in childhood onset idiopathic GHD. In this study, 24 children treated for idiopathic IGHD and in whom GH treatment had been discontinued at the end of linear growth were called back for a reassessment of GH and ACTH secretion. Eleven of 24 patients (45\%) showed a subnormal cortisol response to Synacthen test. Five of these patients had impaired adrenal function in the absence of adult GHD. Cortisol concentrations were lower in adulthood, compared with results obtained in adolescence [19]. Similarly, Di lorgi et al reported development of ACTH Deficiency in $38.5 \%$ of 13 young adults treated in childhood for GHD due to ectopic posterior pituitary and who had a normal GH response to ITT $(5-10 \mu \mathrm{g} / \mathrm{L})$ at retesting [14] and Miyamoto et al documented a gradual evolution of ACTH deficiency over time with suboptimal cortisol peaks in response to ITT in $24 \%$ (before age 10 years) and $56 \%$ (before age 25 years) of patients with prenatal or perinatalonset hypopituitarism and an invisible or thin pituitary stalk [13]. These findings show that there is a potentially increased risk of undiagnosed adrenal insufficiency in patients with idiopathic childhood onset IGHD (even in absence of H-P abnormalities, TSH Deficiency and adult GHD), stressing the need for continuous, regular endocrine evaluation in order to prevent a missed diagnosis of a silently evolving, potentially lethal, adrenal insufficiency [19].

\begin{tabular}{l} 
Manuela Cerbone 9/10/2017 13:00 \\
\hline Deleted: AP \\
\hline Manuela Cerbone 9/10/2017 13:01 \\
\hline Formatted: Font color: Auto \\
\hline Manuela Cerbone 9/10/2017 13:09 \\
\hline Deleted: PSD \\
\hline Manuela Cerbone 9/10/2017 13:07 \\
\hline Deleted: PSIS \\
\hline Manuela Cerbone 9/10/2017 12:58 \\
\hline
\end{tabular}

Deleted: EPP

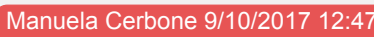

Formatted: Font:Bold, Underline

Manuela Cerbone 9/10/2017 12:47

Formatted: Normal, No bullets or numbering

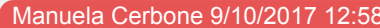

Deleted: EPP

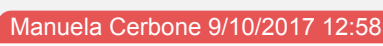

Deleted: EPP

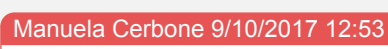

Deleted: PS 
There is some evidence suggesting that GH deficiency masks central hypothyroidism in a significant proportion of hypopituitary patients, and this may be unmasked after commencement of GH replacement. The mechanism of development of central hypothyroidism post-GH remains unclear. The start of GH therapy has been associated with a decrease in FT4 concentrations in the absence of significant paralle changes in FT3 and TSH concentrations. Two mechanisms have been suggested to explain the above discrepancies. The first points to an inhibition of TSH release mediated by an increased somatostatinergic tone directly induced by $\mathrm{GH}$. Indeed, Jorgensen et al documented a significant blunting of the nocturnal TSH surge after the administration of GH to GHD adults [20]. The second proposes an increased extrathyroidal conversion of $\mathrm{T} 4$ to $\mathrm{T} 3$ or an enhancement of $\mathrm{T} 4$ to $\mathrm{T} 3$ deiodination possibly mediated by the generation of IGF-1 [20].

In a study of 20 euthyroid children with idiopathic IGHD and 6 children with CPHD secondary to organic lesions, GH replacement therapy induced central hypothyroidism only in children with CPHD [21]. Stronger evidence was found in a large cohort study of 243 severely GH-deficient adults, whereby $36 \%$ of the previously euthyroid GHD patients developed central hypothyroidism after the start of GH treatment. Additionally $16 \%$ of the patients who were already hypothyroid before the start of GH treatment needed an increase in the L-T4 dose. Patients at higher risk of developing post-GH hypothyroidism included those with a serum T4 concentration towards the lower end of the normal reference range [22]. The results of these studies seem to suggest that $\mathrm{GH}$ treatment unmasks central hypothyroidism mainly in patients with "organic" GHD. However, a recent review concluded with the recommendation that all patients starting GH should have their thyroid function monitored closely, particularly in the first 6 months following the onse of treatment, to identify those who will develop hypothyroidism [23]

\section{Risk factors for progression from IGHD to CPHD}

The risk factors for progression from IGHD to CPHD are listed in Table 2.

\section{Etiology of IGHD (organic vs idiopathic)}

The risk of developing CPHD in children with organic vs idiopathic IGHD has been assessed in a large ( $\mathrm{n}$ : 5805) prospective paediatric study. Children who developed CPHD had more frequently a diagnosis of intracranial tumour or mutations in genes controlling H-P development and/or function as compared with those with idiopathic GHD [4]. Conversely, in a study of 165 IGHD and 3006 CPHD adult patients, progression from IGHD into CPHD was not predicted by the etiology nor by a history of surgery or radiotherapy [15]. Taken together, the results of these two studies suggest that organic GHD is more likely to progress to CPHD than idiopathic GHD; however within the organic cases, the specific etiology (surgery vs radiotherapy vs malignancies) does not predict the risk of progression to CPHD.

\section{Structural abnormalities of the forebrain and H-P region}

Several groups have studied the correlation between H-P congenital abnormalities and development of anterior pituitary hormone deficiencies. An association between specific H-P abnormalities and progression to CPHD has been documented in most, but not all, studies.

In a recent study of 121 children with IGHD ( $n=81)$ and CPHD ( $n=40)$, IGHD patients showing absent pituitary stalk and ectopic posterior pituitary $(n=5)$ inevitably developed additional hormone deficiencies [24]. Similarly, in 83 patients with childhood onset IGHD, pituitary stalk abnormalities were the strongest risk factor ( 3.28 fold) for the development of CPHD [6]. Partially in contrast with previous results, in a study of 31 children with congenital hypopituitarism, empty sella and stalk abnormalities were reported more frequently in CPHD than in the IGHD group, but a hypoplastic pituitary gland, posterior pituitary abnormalities and the MRI tetrad (empty sella, absent/hypoplastic anterior pituitary, absent/redundant stalk, posterior pituitary abnormalities) were more prevalent in IGHD than in CPHD and correlated with the severity of GH deficiency. In this paper imaging abnormalities correlated best with severity of GH deficiency rather than number of hormone deficiencies [25].
Formatted: Font:Underline, Font color: Black

Manuela Cerbone 9/10/2017 12:47

Formatted: Normal, No bullets or numbering

Manuela Cerbone 9/10/2017 12:47

Formatted: Underline

Manuela Cerbone 9/10/2017 12:47

Formatted: Normal, No bullets or numbering

Manuela Cerbone 9/10/2017 13:01

Deleted: AP

Manuela Cerbone 9/10/2017 13:07

Deleted: PSA

Manuela Cerbone 9/10/2017 12:58

Deleted: EPP

Manuela Cerbone 9/10/2017 12:53

Deleted: PS

Manuela Cerbone 9/10/2017 13:03

Deleted: PP

Manuela Cerbone 9/10/2017 13:01

Deleted: AP

Manuela Cerbone 9/10/2017 13:03

Deleted: PP 
Several other authors have focused on the association between stalk abnormalities and the risk of developing CPHD. The first study documenting a strong association between absent pituitary stalk after gadolinium injection and CPHD was performed on 18 children ( 8 with IGHD and 10 with CPHD) with an unidentified pituitary stalk at first MRI, who were then re-evaluated with MRI using gadopentetate dimeglumine (Gd-DTPA). After Gd-DTPA, patients with absent pituitary stalk had a risk of developing CPHD 27 times greater than had those with an identified pituitary stalk. The vascular component of the pituitary stalk was easily recognized after Gd-DTPA in most IGHD patients, but exceptionally in CPHD, whereas the neural component was lacking regardless of whether patients had IGHD or CPHD [26]. In a recent study comparing 39 patients with CPHD with 46 patients with IGHD, all patients whose infundibulum was not visualized after Gd-DTPA injection belonged to the CPHD group [27]. Similar results were found in another study of 25 children with GHD and ectopic posterior pituitary divided in two groups based on the visibility (Group 1) or absence (Group 2) of the after gadolinium injection. Most patients in group 1 (12 of 14 cases) showed IGHD; on the other hand, all but one patient in group 2 showed CPHD. Additionally, an ectopic posterior pituitary at the level of the median eminence was found in all group 2 patients and in 6 of the 14 group 1 patients, whereas the ectopic posterior pituitary, was located at various points along the pituitary stalk in the other 8 patients within group 1 . The height of the adenohypophysis was smaller in group 2 , and when correlated with age and pubertal status, the prevalence of anterior pituitary hypoplasia was also higher in group 2. Although the pathogenesis of GH deficiency with ectopic posterior pituitary remains unclear, these results suggest that in cases of GHD associated with ectopic posterior pituitary, patients with no visible pituitary stalk on MRI after gadolinium injection present a more severe form of the disease with CPHD, whereas visibility of the pituitary stalk is associated with IGHD. The results of the study also show that posterior pituitary location along the stalk is a significant protective factor for the severity of hormonal phenotype, with a greater number of hormone deficiencies present when the posterior lobe is localized at the median eminence or in the hypothalamic region [28]. In contrast to these previous studies, a recent study of 26 patients with partial or complete absence of pituitary stalk associated with anterior pituitary hypoplasia or ectopic posterior pituitary failed to document a significant association between hormonal dysfunction and pituitary stalk visibility or the location of the ectopic posterior pituitary [12].

It is well known that midline brain abnormalities and optic nerve hypoplasia, in addition to defects in the $\mathrm{H}$ $\mathrm{P}$ region, are also associated with a higher risk of developing hypopituitarism. Patients with septo-optic dysplasia have been found to present with some degree of hypopituitarism in $50-66 \%$ of cases [29-34]. In a retrospective study of MRI findings and endocrine function on 170 children with or 'at-risk' (optic nerve hypoplasia, holoprosencephaly or multiple midline systemic defects) of congenital hypopituitarism, the risk of hypopituitarism was 27 times greater in patients with ectopic posterior pituitary, Anterior pituitary hypoplasia and pituitary stalk absence were also significantly associated with hypopituitarism. With respect to the type or severity of hypopituitarism, CPHD was more often associated with an abnormal corpus callosum and stalk abnormalities. Overall, midline forebrain defects were 5.2 times more prevalent in patients with CPHD as compared with IGHD [34]. The result of this study confirm that midline forebrain defects are associated with a higher risk of CPHD, but also suggest that within children at risk of developing hypopituitarism due to the presence of optic nerve hypoplasia and/or midline brain abnormalities, corpus callosum and pituitary stalk abnormalities are strong predictors of CPHD.

To summarize, HP, ON and midline brain abnormalities have been reported more frequently in children with CPHD compared to IGHD. absent pituitary stalk, particularly in its vascular component, seems to be the strongest predictor of the development of additional pituitary deficits in IGHD. An ectopic posterior pituitary, especially when located at the median eminence or the hypothalamic level, has been strongly associated with development of additional pituitary deficits. Corpus callosum abnormalities are associated with CPHD, whereas anterior pituitary hypoplasia can be found both in IGHD and CPHD. However, an empty sella is more likely to be associated with CPHD and the degree of hypoplasia of the adenohypophysis has been associated with the number and severity of pituitary deficits. Regarding the type of pituitary deficits,

H-P abnormalities (ectopic posterior pituitary and absent pituitary stalk) are more frequently found in association with ACTH and TSH than FSH/LH deficiency.

Genetic defects

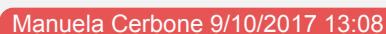

Deleted: PSA...after gadolinium injec ... [1]

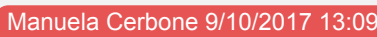

Deleted: $(\mathrm{ONH}), \ldots \mathrm{n}$ addition to defe $\ldots$... [2]

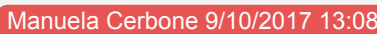

Deleted: PSA... particularly in its vasc ... [3]

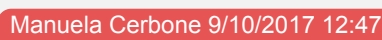

Deleted:

Manuela Cerbone 9/10/2017 12:47

Formatted: Font:Underline, Font color:

Formatte

Manuela Cerbone 9/10/2017 12:47

Formatted: Normal, No bullets or

numbering 
IGHD can present as a result of defects in genes involved in pituitary development or in the GH secretory pathway. The past decades have witnessed an explosion in our understanding of the development of the | anterior pituitary gland and of mechanisms that underlie the diagnosis of GHD and CPHD. However, to date, genetic mutations have been identified only in a modest proportion of patients. It is clear that many genes remain to be identified and the characterization of these will further elucidate the pathogenesis of these complex conditions [35].

Transcription factors such as HESX1, PROP1, POU1F1, LHX3, LHX4, SOX2 and SOX3 are involved in the etiology of CPHD and they are associated with highly variable phenotypes encompassing IGHD, CPHD, or

more complex disorders such as septo-optic dysplasia [35-38]. PROP1 mutations constitute the most common genetic cause for $\operatorname{CPHD}([9,39,40]$ [41]) with a poor genotype-phenotype correlation and different pattern of evolution of endocrinopathies ([42],[12]).

Mutation in $\mathrm{GH} 1$ had been historically associated with isolated congenital GHD. However evolution of additional deficits including ACTH, TSH, PRL, LH/FSH has been subsequently documented [38]. In a study of 57 subjects belonging to 19 families harbouring different splice site as well as missense mutations within $\mathrm{GH}-1$, subjects presenting with a splice site mutation within the first 2 bp of intervening sequence 3 ( $5^{\prime}$ IVS + $1 /+2 \mathrm{bp}$ ) leading to a skipping of exon 3 were found to be more likely to develop other pituitary hormone deficiencies, mainly ACTH and TSH. In addition, although the patients with missense mutations had previously been reported to be less affected, a number of patients presenting with the P89L missense GH1 form showed some additional pituitary hormone impairment. The development of multiple hormonal deficiencies was not age-dependent, and there was a great variability in onset, severity, and progression, even within families with the same mutation [43]. A recent paper has also described evolution of gonadotropin deficiency in one adult treated for childhood onset IGHD due to an autosomal dominant splicing mutation in $\mathrm{GH}-1$ (intervening sequence-3 $+1 \mathrm{G}>\mathrm{A}$ ) [44]. The message of clinical importance from the previous studies is that the pituitary endocrine status of patients with GH1 mutations should continue to be monitored closely over the years because further hormonal deficiencies may evolve with time.

Taken together, the results of the above reported studies show that, in congenital forms of IGHD, the presence of certain genetic defects in transcription factors involved in $\mathrm{H}-\mathrm{P}$ development or in $\mathrm{GH}$ secretion can predispose to a higher risk of developing additional pituitary deficits. However the genotype-phenotype correlation is poor and the endocrine phenotype is highly variable in these patients.

\section{Duration of follow-up}

In a large prospective study of 5805 pediatric patients with idiopathic IGHD, the duration of follow-up was the strongest predictor of evolving endocrinopathies [4]. A similar correlation was found in 716 children with organic IGHD from the same database [5]. Hence, long-term monitoring of pituitary function is recommended, irrespective of the etiology of GHD.

\section{Severity of GHD}

In the study by Blum et al., predictors of evolution of endocrinopathy in IGHD patients included a more severe GHD phenotype, as reflected by lower baseline height SDS and peak stimulated GH; children who developed CPHD also had a better first-year response to GH therapy, in keeping with the more severe GHD. However, importantly, the presence of milder forms of GHD did not appear to exclude the future development of CPHD: ten patients who developed CPHD were initially diagnosed as having GH neurosecretory dysfunction and 20 patients had peak $\mathrm{GH}$ concentrations above the generally used diagnostic cut-off for GHD of $10 \mu \mathrm{g} / \mathrm{l}$ [4]. A correlation between the severity of GHD and the risk of developing additional pituitary deficiencies has also been documented in children with organic GHD [5]. The results of these studies suggest that children with severe IGHD display a higher risk of developing CPHD irrespective of the underlying etiology. However, the presence of milder forms of GHD does not exclude the future development of CPHD.

\section{Gender:}

In two studies female gender was a predictor of CPHD development in children with IGHD [5, Otto Pituitary $2015,6]$.

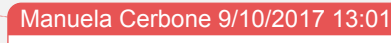

Deleted: AP

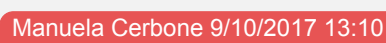

Deleted: SOD

\begin{tabular}{|c|}
\hline $\begin{array}{l}\text { Manuela Cerbone 9/10/2017 12:47 } \\
\text { Deleted: }\end{array}$ \\
\hline Manuela Cerbone 9/10/2017 12:47 \\
\hline Formatted: Underline \\
\hline Manuela Cerbone 9/10/2017 12:47 \\
\hline $\begin{array}{l}\text { Formatted: Normal, No bullets or } \\
\text { numbering }\end{array}$ \\
\hline Manuela Cerbone 9/10/2017 12:47 \\
\hline Deleted: \\
\hline Manuela Cerbone 9/10/2017 12:47 \\
\hline Formatted: Underline \\
\hline Manuela Cerbone 9/10/2017 12:47 \\
\hline $\begin{array}{l}\text { Formatted: Normal, No bullets or } \\
\text { numbering }\end{array}$ \\
\hline Manuela Cerbone 9/10/2017 12:48 \\
\hline Deleted: \\
\hline Manuela Cerbone 9/10/2017 12:48 \\
\hline Formatted: Font:Underline \\
\hline Manuela Cerbone 9/10/2017 12:48 \\
\hline $\begin{array}{l}\text { Formatted: Normal, No bullets or } \\
\text { numbering }\end{array}$ \\
\hline
\end{tabular}


Perinatal/neonatal history

In a large prospective study of children with idiopathic IGHD, delivery complications, breech delivery, and perinatal/neonatal adverse events had occurred more frequently in patients who developed CPHD [4]. In another study of 31 children with congenital hypopituitarism, $78 \%$ of children in the breech delivery group, $70 \%$ in the vertex group and 50\% in the lower segment caesarian section group had CPHD [25]. Conversely, I in a retrospective and prospective study of 26 patients with pituitary stalk dysgenesis, no significant association was found between hormonal dysfunction and previous perinatal damage or breech delivery [12]. To conclude, an association between the evolution of endocrinopathy and delivery complications, breech delivery, and perinatal/neonatal adverse events has been found in some, but not all, studies.

Extra-pituitary malformations,

In a study of a cohort of pituitary stalk interruption syndrome, patients comparing subgroups with or without extra-pituitary malformations, CPHD was observed significantly more often in patients with, compared with those without, extra-pituitary malformations ( 87.5 vs $69.5 \%$ respectively). Extra-pituitary malformations included CNS, ocular, dental, craniofacial, sella turcica, or cardiac malformations [11].

\section{Conclusions:}

Children with IGHD display a significant risk of developing additional pituitary deficiencies. This risk ranges between $5.5 \%$ in childhood-onset idiopathic IGHD and $35 \%$ in adult-onset organic IGHD. H-P, optic nerves and midline brain abnormalities predispose to a higher risk of developing additional pituitary deficits (up to $45-100 \%$ in children with pituitary stalk interruption syndrome). Absent pituitary stalk after gadolinium administration and an ectopic posterior pituitary at the median eminence and hypothalamic level are highly predictive of CPHD. Empty sella, absent/hypoplastic corpus callosum and optic nerve hypoplasia are also associated with a higher risk of evolution of endocrinopathy. The type and age at occurrence of additional pituitary deficits are highly variable and they cannot be easily predicted based on the etiology, severity and age at onset of IGHD. Importantly, idiopathic IGHD without structural H-P abnormalities does not exclude the occurrence of additional pituitary deficits. A longer duration of follow-up is associated with a higher risk of additional pituitary deficits, suggesting that hypopituitarism is a dynamic condition where new deficiencies can appear years after the initial diagnosis. Adrenal insufficiency can silently evolve years after the onset of GHD and the start of GH treatment in patients with GHD can unmask central hypothyroidism. To conclude, the current available evidence supports longstanding recommendations for the need, in al patients diagnosed with IGHD, of a careful and indefinite follow-up for additional pituitary hormone deficiencies, irrespective of the age at presentation, severity, etiology and of the reversibility of GHD in adolescence [2].

\section{$\underline{\text { References }}$}

1. Molitch, M.E., et al., Evaluation and treatment of adult growth hormone deficiency: an Endocrine Society clinical practice guideline. J Clin Endocrinol Metab, 2011. 96(6): p. 1587-609.

2. Consensus guidelines for the diagnosis and treatment of growth hormone (GH) deficiency in childhood and adolescence: summary statement of the GH Research Society. GH Research Society. J Clin Endocrinol Metab, 2000. 85(11): p. 3990-3.

3. Ho, K.K., Consensus guidelines for the diagnosis and treatment of adults with GH deficiency II: a statement of the GH Research Society in association with the European Society for Pediatric Endocrinology, Lawson Wilkins Society, European Society of Endocrinology, Japan Endocrine Society, and Endocrine Society of Australia. Eur J Endocrinol, 2007. 157(6): p. 695-700.

4. Blum, W.F., et al., Development of additional pituitary hormone deficiencies in pediatric patients originally diagnosed with idiopathic isolated GH deficiency. Eur J Endocrinol, 2014. 170(1): p. 13-21.

5. Child, C.J., et al., Development of additional pituitary hormone deficiencies in pediatric patients originally diagnosed with isolated growth hormone deficiency due to organic causes. Eur J Endocrinol, 2016. 174(5): p. 669-79.
Manuela Cerbone 9/10/2017 12:48

Deleted:

Manuela Cerbone 9/10/2017 12:48

Formatted: Underline

Manuela Cerbone 9/10/2017 12:48

Formatted: Normal, No bullets or numbering

Manuela Cerbone 9/10/2017 13:09

Deleted: PSD

Manuela Cerbone 9/10/2017 12:48

Deleted:

Manuela Cerbone 9/10/2017 13:05

Deleted: (EPM)

Manuela Cerbone 9/10/2017 12:48

Formatted: Underline

Manuela Cerbone 9/10/2017 12:48

Formatted: Normal, No bullets or numbering

Manuela Cerbone 9/10/2017 13:07

Deleted: PSIS

Manuela Cerbone 9/10/2017 13:05

Formatted: No underline

Manuela Cerbone 9/10/2017 13:05

Deleted: EPM

Manuela Cerbone 9/10/2017 13:05

Deleted: EPM

Manuela Cerbone 9/10/2017 13:05

Deleted: EPM

Manuela Cerbone 9/10/2017 12:48

Formatted: Font:Bold, Underline

Manuela Cerbone 9/10/2017 12:48

Formatted: Normal, No bullets or numbering

Manuela Cerbone 9/10/2017 13:07

Deleted: PSIS

Manuela Cerbone 9/10/2017 13:08

Deleted: PSA

Manuela Cerbone 9/10/2017 12:59

Deleted: EPP 
6. Otto, A.P., et al., Frequent development of combined pituitary hormone deficiency in patients initially diagnosed as isolated growth hormone deficiency: a long term follow-up of patients from a single center. Pituitary, 2015. 18(4): p. 561-7.

7. Deal, C., et al., Associations between pituitary imaging abnormalities and clinical and biochemical phenotypes in children with congenital growth hormone deficiency: data from an international observational study. Horm Res Paediatr, 2013. 79(5): p. 283-92.

8. Jagtap, V.S., et al., Ectopic posterior pituitary and stalk abnormality predicts severity and coexisting hormone deficiencies in patients with congenital growth hormone deficiency. Pituitary, 2012. 15(2): p. 243-50.

9. Bar, C., et al., Pituitary Stalk Interruption Syndrome from Infancy to Adulthood: Clinical, Hormonal, and Radiological Assessment According to the Initial Presentation. PLoS One, 2015. 10(11): p e0142354.

10. Tauber, M., et al., Long-term evolution of endocrine disorders and effect of GH therapy in 35 patients with pituitary stalk interruption syndrome. Horm Res, 2005. 64(6): p. 266-73.

11. Reynaud, R., et al., Pituitary stalk interruption syndrome in 83 patients: novel HESX1 mutation and severe hormonal prognosis in malformative forms. Eur J Endocrinol, 2011. 164(4): p. 457-65.

12. Fernandez-Rodriguez, E., et al., Pituitary stalk dysgenesis-induced hypopituitarism in adult patients: prevalence, evolution of hormone dysfunction and genetic analysis. Neuroendocrinology, 2011 93(3): p. 181-8.

13. Miyamoto, J., et al., Development of growth hormone and adrenocorticotropic hormone deficiencies in patients with prenatal or perinatal-onset hypothalamic hypopituitarism having invisible or thin pituitary stalk on magnetic resonance imaging. Endocr J, 2001. 48(3): p. 355-62.

14. di lorgi, N., et al., Deterioration of growth hormone (GH) response and anterior pituitary function in young adults with childhood-onset GH deficiency and ectopic posterior pituitary: a two-year prospective follow-up study. J Clin Endocrinol Metab, 2007. 92(10): p. 3875-84.

15. Klose, M., et al., From isolated GH deficiency to multiple pituitary hormone deficiency: an evolving continuum - a KIMS analysis. Eur J Endocrinol, 2009. 161 Suppl 1: p. S75-83.

16. Walvoord, E.C., M.B. Rosenman, and E.A. Eugster, Prevalence of adrenocorticotropin deficiency in children with idiopathic growth hormone deficiency. J Clin Endocrinol Metab, 2004. 89(10): p. 50304.

17. Makino, S., et al., Late onset of adrenocortical failure in GH deficiency with invisible pituitary stalk: a case report of a 48-year-old Japanese man and review of the literature. Endocr J, 2002. 49(2): p. 231-40.

18. Maghnie, M., et al., Hypothalamic-pituitary dysfunction in growth hormone-deficient patients with pituitary abnormalities. J Clin Endocrinol Metab, 1991. 73(1): p. 79-83.

19. Lange, M., et al., High risk of adrenal insufficiency in adults previously treated for idiopathic childhood onset growth hormone deficiency. J Clin Endocrinol Metab, 2003. 88(12): p. 5784-9.

20. Jorgensen, J.O., et al., Effects of growth hormone therapy on thyroid function of growth hormonedeficient adults with and without concomitant thyroxine-substituted central hypothyroidism. J Clin Endocrinol Metab, 1989. 69(6): p. 1127-32.

21. Giavoli, C., et al., Recombinant $h G H$ replacement therapy and the hypothalamus-pituitary-thyroid axis in children with GH deficiency: when should we be concerned about the occurrence of central hypothyroidism? Clin Endocrinol (Oxf), 2003. 59(6): p. 806-10.

22. Agha, A., et al., Unmasking of central hypothyroidism following growth hormone replacement in adult hypopituitary patients. Clin Endocrinol (Oxf), 2007. 66(1): p. 72-7.

23. Behan, L.A., J.P. Monson, and A. Agha, The interaction between growth hormone and the thyroid axis in hypopituitary patients. Clin Endocrinol (Oxf), 2011. 74(3): p. 281-8.

24. Bozzola, M., et al., Long-term follow-up evaluation of magnetic resonance imaging in the prognosis of permanent GH deficiency. Eur J Endocrinol, 2000. 143(4): p. 493-6.

25. Dutta, P., et al., Congenital hypopituitarism: clinico-radiological correlation. J Pediatr Endocrinol Metab, 2009. 22(10): p. 921-8. 
26. Maghnie, M., et al., Dynamic MRI in the congenital agenesis of the neural pituitary stalk syndrome: the role of the vascular pituitary stalk in predicting residual anterior pituitary function. Clin Endocrinol (Oxf), 1996. 45(3): p. 281-90.

27. Kandemir, N., et al., Magnetic resonance imaging in growth hormone deficiency: relationship between endocrine function and morphological findings. J Pediatr Endocrinol Metab, 2000. 13(2): p. 171-8.

28. Chen, S., et al., Growth hormone deficiency with ectopic neurohypophysis: anatomical variations and relationship between the visibility of the pituitary stalk asserted by magnetic resonance imaging and anterior pituitary function. J Clin Endocrinol Metab, 1999. 84(7): p. 2408-13.

29. Atapattu, N., et al., Septo-optic dysplasia: antenatal risk factors and clinical features in a regional study. Horm Res Paediatr, 2012. 78(2): p. 81-7.

30. Riedl, S., et al., Refining clinical phenotypes in septo-optic dysplasia based on MRI findings. Eur J Pediatr, 2008. 167(11): p. 1269-76.

31. Mehta, A. and M.T. Dattani, Developmental disorders of the hypothalamus and pituitary gland associated with congenital hypopituitarism. Best Pract Res Clin Endocrinol Metab, 2008. 22(1): p. 191-206.

32. Kelberman, D. and M.T. Dattani, Genetics of septo-optic dysplasia. Pituitary, 2007. 10(4): p. 393407.

33. Birkebaek, N.H., et al., Endocrine status in patients with optic nerve hypoplasia: relationship to midline central nervous system abnormalities and appearance of the hypothalamic-pituitary axis on magnetic resonance imaging. J Clin Endocrinol Metab, 2003. 88(11): p. 5281-6.

34. Mehta, A., et al., Congenital hypopituitarism: clinical, molecular and neuroradiological correlates. Clin Endocrinol (Oxf), 2009. 71(3): p. 376-82

35. Dattani, M.T., Growth hormone deficiency and combined pituitary hormone deficiency: does the genotype matter? Clin Endocrinol (Oxf), 2005. 63(2): p. 121-30.

36. Kelberman, D. and M.T. Dattani, Role of transcription factors in midline central nervous system and pituitary defects. Endocr Dev, 2009. 14: p. 67-82.

37. Kelberman, D., et al., Genetic regulation of pituitary gland development in human and mouse. Endocr Rev, 2009. 30(7): p. 790-829.

38. Alatzoglou, K.S., et al., Isolated growth hormone deficiency (GHD) in childhood and adolescence: recent advances. Endocr Rev, 2014. 35(3): p. 376-432.

39. Rohayem, J., et al., Long-Term Outcomes, Genetics, and Pituitary Morphology in Patients with Isolated Growth Hormone Deficiency and Multiple Pituitary Hormone Deficiencies: A Single-Centre Experience of Four Decades of Growth Hormone Replacement. Horm Res Paediatr, 2016. 86(2): p. 106-116.

40. Deladoey, J., et al., "Hot spot" in the PROP1 gene responsible for combined pituitary hormone deficiency. J Clin Endocrinol Metab, 1999. 84(5): p. 1645-50

41. Kelberman, D., et al., Molecular analysis of novel PROP1 mutations associated with combined pituitary hormone deficiency (CPHD). Clin Endocrinol (Oxf), 2009. 70(1): p. 96-103.

42. Fluck, C., et al., Phenotypic variability in familial combined pituitary hormone deficiency caused by a PROP1 gene mutation resulting in the substitution of Arg-->Cys at codon 120 (R120C). J Clin Endocrinol Metab, 1998. 83(10): p. 3727-34.

43. Mullis, P.E., et al., Isolated autosomal dominant growth hormone deficiency: an evolving pituitary deficit? A multicenter follow-up study. J Clin Endocrinol Metab, 2005. 90(4): p. 2089-96.

44. Turton, J.P., et al., Evolution of gonadotropin deficiency in a patient with type II autosomal dominant GH deficiency. Eur J Endocrinol, 2006. 155(6): p. 793-9. 